Erratum

\title{
Erratum to "Gastroenteropancreatic Neuroendocrine Neoplasia Characterization in Portugal: Results from the NETs Study Group of the Portuguese Society of Endocrinology, Diabetes and Metabolism"
}

\author{
A. P. Santos $\mathbb{D}^{1},{ }^{1}$ J. Vinagre $\mathbb{D}^{2,},{ }^{2,3,4}$ P. Soares $\mathbb{D},{ }^{2,3,5}$ I. Claro, ${ }^{6}$ A. C. Sanches, ${ }^{1}$ L. Gomes, ${ }^{7}$ \\ I. Fernandes, ${ }^{8,9}$ A. L. Catarino, ${ }^{10}$ J. Preto, ${ }^{4,5}$ B. D. Pereira, ${ }^{11}$ A. P. Marques, ${ }^{12}$ F. Rodrigues, ${ }^{13}$ \\ C. Amaral, ${ }^{14}$ G. Rocha, ${ }^{15}$ J. C. Mellidez, ${ }^{16}$ H. Simões, ${ }^{17}$ J. M. Lopes $\mathbb{D},{ }^{2,3,4,5}$ M. J. Bugalho $\mathbb{D},{ }^{8,9}$ \\ and On behalf of the NETs Study Group of the Portuguese Society of \\ Endocrinology, Diabetes and Metabolism ${ }^{18}$

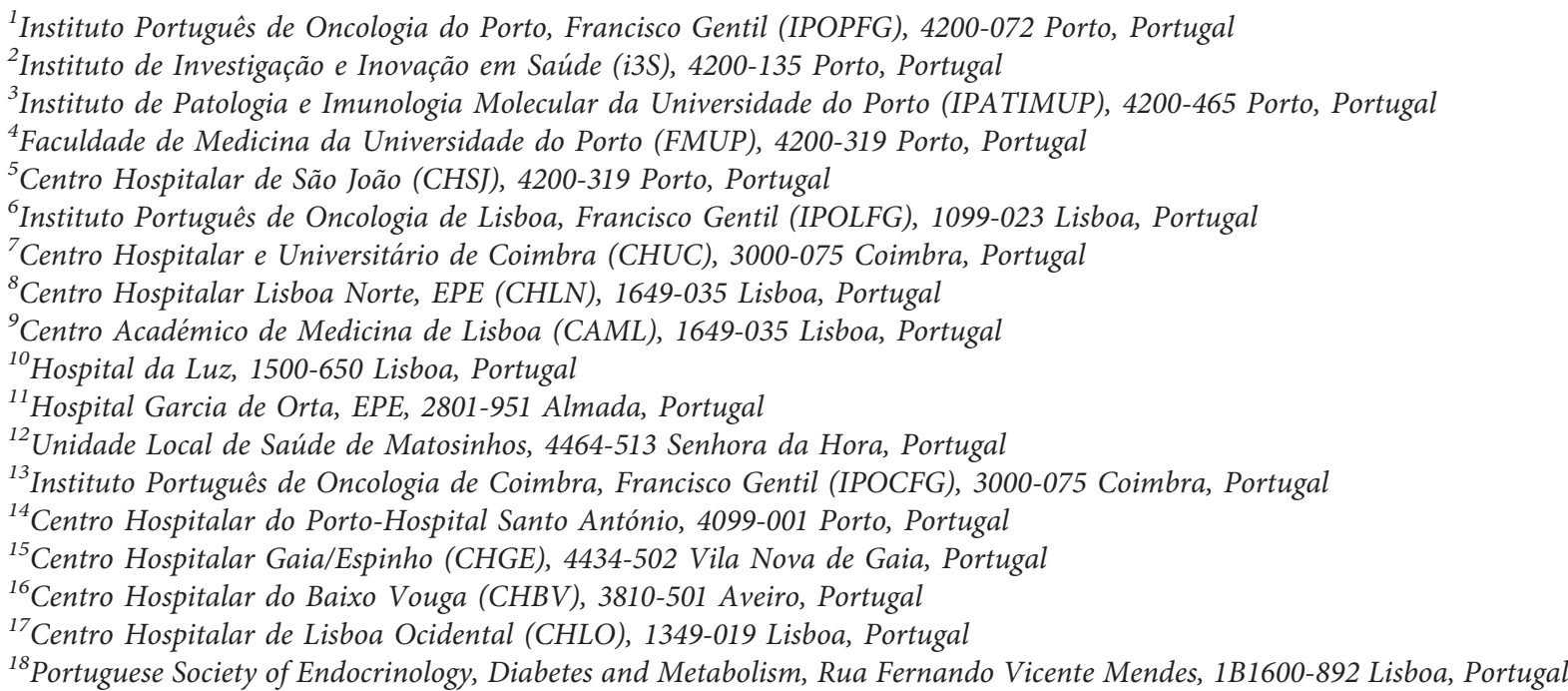 \\ Correspondence should be addressed to A. P. Santos; anapaulasantos@ipoporto.min-saude.pt
}

Received 21 April 2020; Accepted 21 April 2020; Published 18 June 2020

Copyright (C) 2020 A. P. Santos et al. This is an open access article distributed under the Creative Commons Attribution License, which permits unrestricted use, distribution, and reproduction in any medium, provided the original work is properly cited.

In the article titled "Gastroenteropancreatic Neuroendocrine Neoplasia Characterization in Portugal: Results from the NETs Study Group of the Portuguese Society of Endocrinology, Diabetes and Metabolism" [1], the affiliation for I. Claro was labeled incorrectly. The correct affiliation of the author I. Claro is Instituto Português de Oncologia de Lisboa, Francisco Gentil (IPOLFG), 1099-023 Lisboa, Portugal.

\section{References}

[1] A. P. Santos, J. Vinagre, P. Soares et al., "Gastroenteropancreatic neuroendocrine neoplasia characterization in Portugal: results from the NETs study group of the Portuguese society of endocrinology, diabetes and metabolism," International Journal of Endocrinology, Article ID 4518742, 10 pages, 2019. 